Supporting Information Kirsch, Overman, Watson

\title{
Monomeric Cobalt Oxazoline Palladacycles (COP). Useful Catalysts for Catalytic Asymmetric Rearrangement of Allylic Trichloroacetimidates
}

Stefan F. Kirsch, Larry E. Overman*, Mary P. Watson

Department of Chemistry, 516 Rowland Hall, University of California, Irvine, CA 92697-2025

\section{Supporting Information}

Copies of HPLC and GC traces used to determine enantiopurity of rearranged products; copies of ${ }^{1} \mathrm{H}$ and ${ }^{13} \mathrm{C}$ NMR spectra for $2, \mathbf{3}, \mathbf{5}$ and $\mathbf{8} .{ }^{1}$

(17 Pages)

General experimental details: All HPLC and GC analyses used to determine enantiomeric purity were calibrated with samples of the racemate.

${ }^{1}$ Compounds 5 and 8 are known compounds: Anderson, C. E., Overman, L. E. J. Am. Chem. Soc. 2003, 125, 12412-12413. 


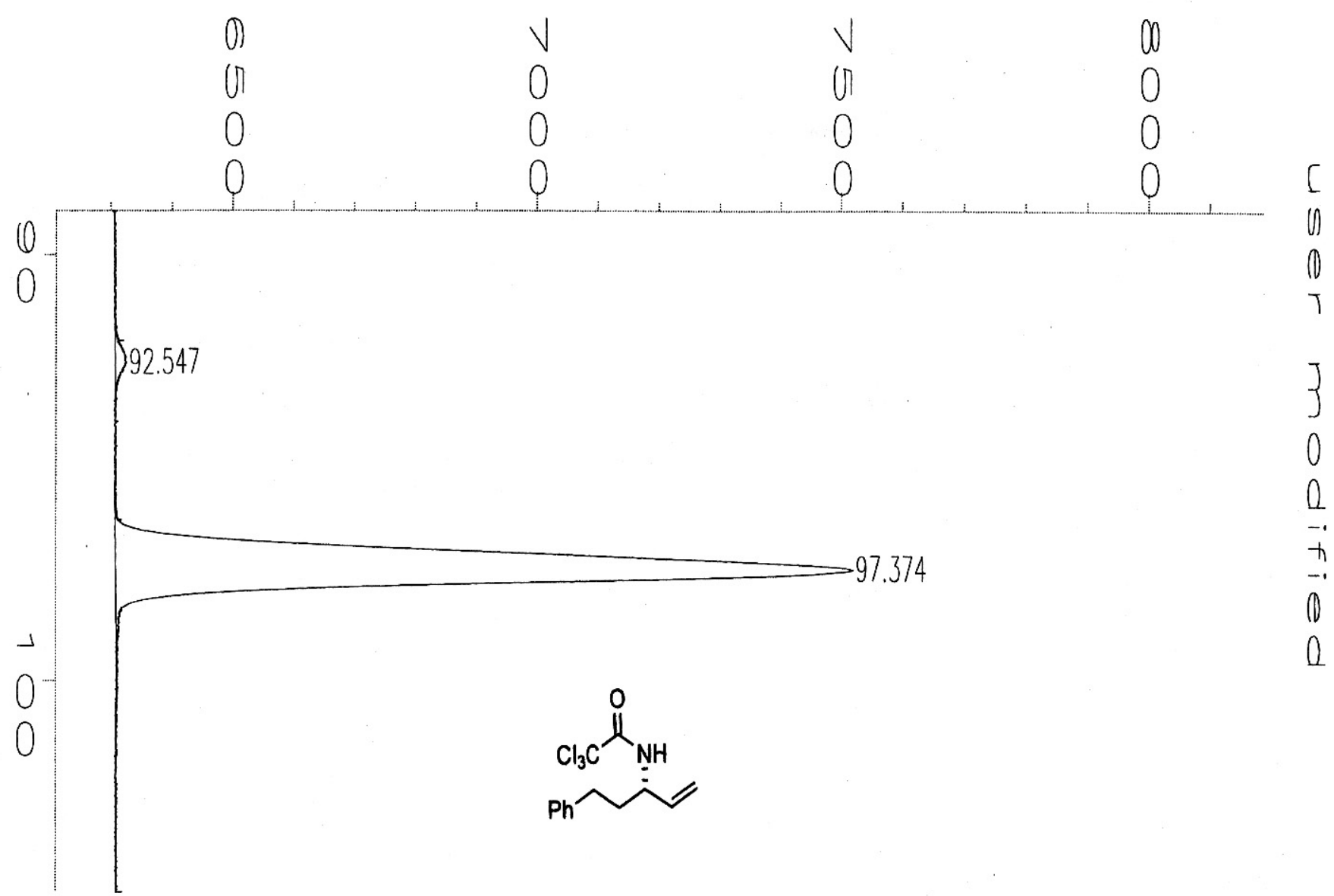

Area Percent Report

ta File Name : C: \HPCHEM $\backslash 2 \backslash$ DATA $\backslash S T E F A N \backslash S K 142 D . D$

\begin{tabular}{|c|c|c|}
\hline unerator & : MARY & \\
\hline Instrument & : INSTRUMEN & \\
\hline Sample Name & : SK142D & \\
\hline Run Time Bar Code: & & \\
\hline Acquired on : & : 18 Mar 04 & $05: 31 \mathrm{PM}$ \\
\hline Report Created on: & 1: 18 Mar 04 & $09: 52$ \\
\hline
\end{tabular}

Page Number : 1

Vial Number :

Injection Number:

Sequence Line :

Instrument Method: LONGRUN.MTH

Analysis Method : LONGRUN.MTH

Sig. 2 in $C: \backslash H P C H E M \backslash 2 \backslash D A T A \backslash S T E F A N \backslash S K 142 D . D$

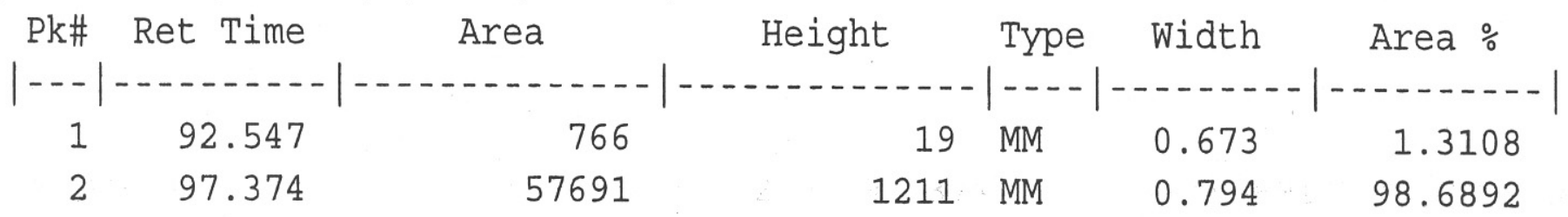

Total area $=58457$

User Modified

(Chiraldex Gamma Cyclodextrin Trifluoroacetyl, $20 \mathrm{~m} \times 0.25 \mathrm{~mm}$; initial temperature: $50{ }^{\circ} \mathrm{C}$, final temperature: $\left.150^{\circ} \mathrm{C}, 5^{\circ} \mathrm{C} / \mathrm{min}\right)$ 
$0.5 \%$ IPA in hexanes, odh, $0.7 \mathrm{ml} / \mathrm{min}$
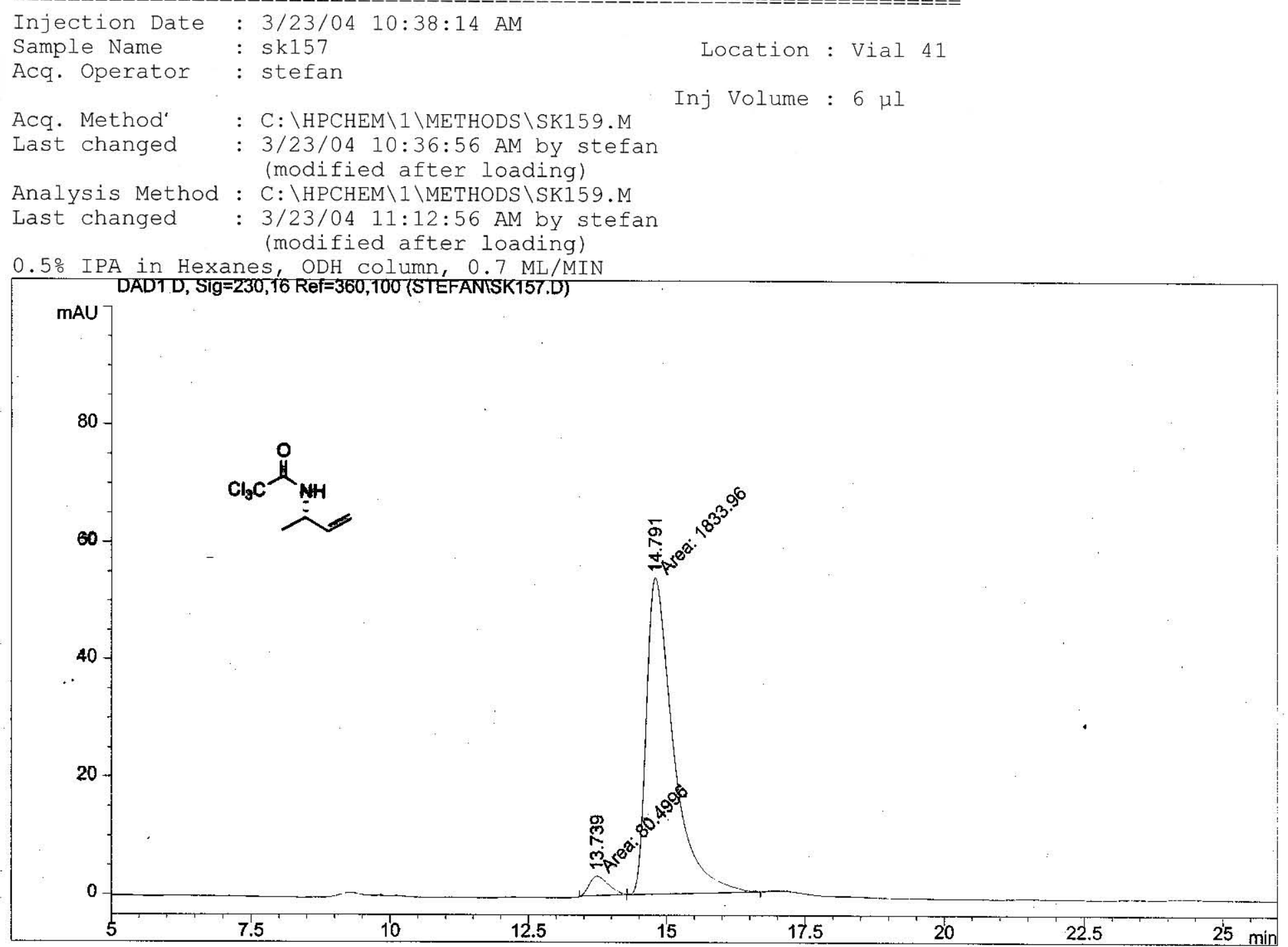

Area Percent Report

$\begin{array}{lll}\text { Sorted By } & : & \text { Signal } \\ \text { Multiplier } & : & 1.0000 \\ \text { Dilution } & : & 1.0000\end{array}$

Use Multiplier \& Dilution Factor with ISTDs

Signal 1: DAD1 D, Sig=230,16 Ref=360, 100

\begin{tabular}{|c|c|c|c|c|c|c|}
\hline $\begin{array}{c}\text { Peak } \\
\quad \#\end{array}$ & $\begin{array}{l}\text { RetTime } \\
\text { [min] }\end{array}$ & Type & $\begin{array}{l}\text { Width } \\
\text { [min] }\end{array}$ & $\begin{array}{c}\text { Area } \\
{\left[\mathrm{mAU}^{*} \mathrm{~S}\right]}\end{array}$ & $\begin{array}{l}\text { Height } \\
\text { [mAU] }\end{array}$ & $\begin{array}{c}\text { Area } \\
\quad \circ\end{array}$ \\
\hline $\begin{array}{l}1 \\
2\end{array}$ & $\begin{array}{l}13.739 \\
14.791\end{array}$ & $\begin{array}{l}\text { MM } \\
\text { MM }\end{array}$ & $\begin{array}{l}0.3999 \\
0.5663\end{array}$ & $\begin{array}{r}80.49957 \\
1833.96484\end{array}$ & $\begin{array}{r}3.35506 \\
53.97146\end{array}$ & $\begin{array}{r}4.2048 \\
95.7952\end{array}$ \\
\hline
\end{tabular}

Totals : $\quad 1914.46441 \quad 57.32652$

Results obtained with enhanced integrator!

Summed Peaks Report 


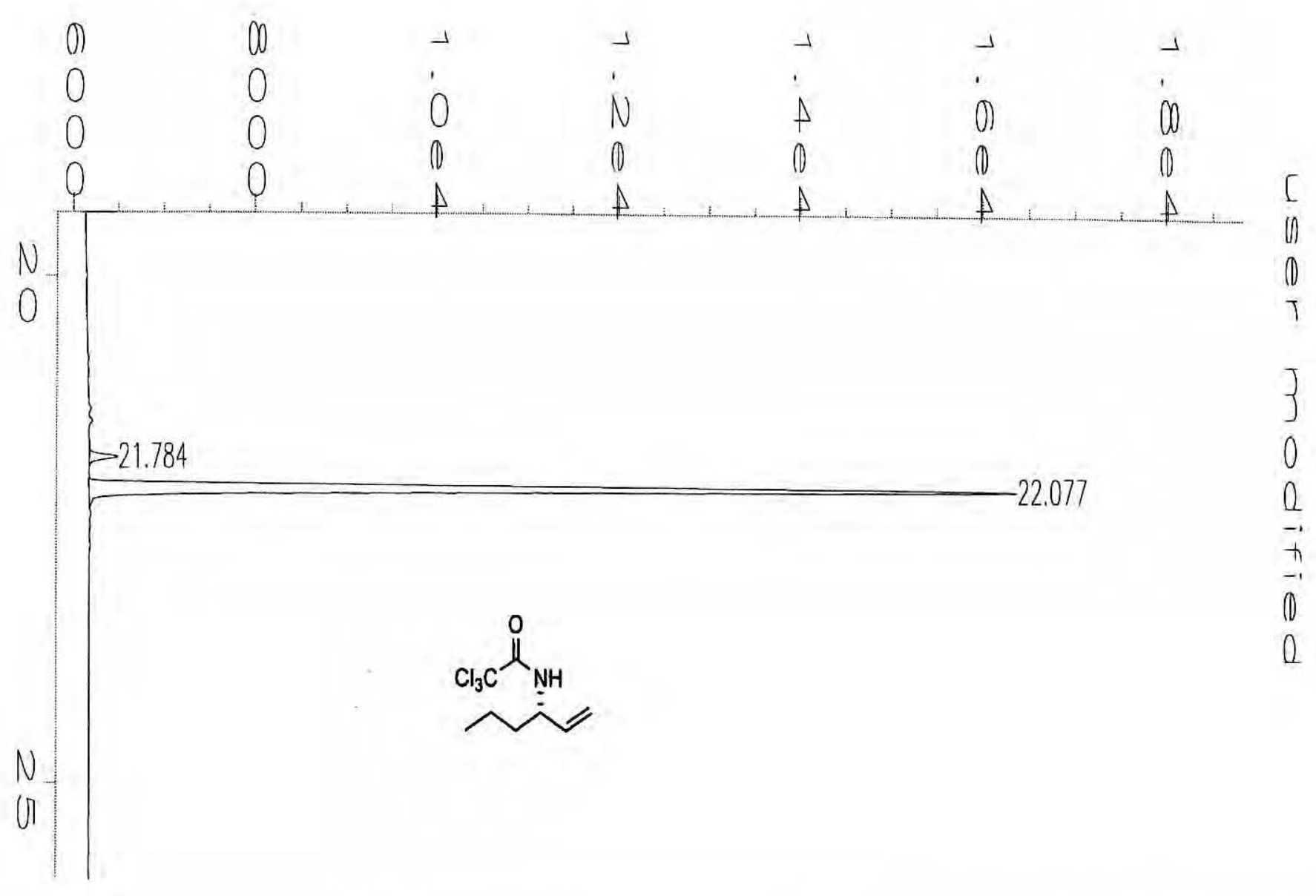

Area Percent Report

Page Number : 1

Vial Number :

Injection Number :

Sequence Line :

Instrument Method: LONGRUN.MTH Analysis Method : LONGRUN.MTH

Sig. 2 in C: $\backslash$ HPCHEM $\backslash 2 \backslash D A T A \backslash K I R S C H 2 \backslash S K 172 . D$

\begin{tabular}{|ccccccc} 
Pk\# & Ret Time & Area & Height & Type & Width & Area $\%$ \\
\hline 1 & 21.784 & 1108 & 314 & MM & 0.059 & 2.6239 \\
2 & 22.077 & 41112 & 10208 & MM & 0.067 & 97.3761
\end{tabular}

Total area $=42219$

User Modified

(Chiraldex Gamma Cyclodextrin Trifluoroacetyl, $20 \mathrm{~m} \times 0.25 \mathrm{~mm}$; initial temperature: $50^{\circ} \mathrm{C}$, final temperature: $150^{\circ} \mathrm{C}, 5^{\circ} \mathrm{C} / \mathrm{min}$ ) 


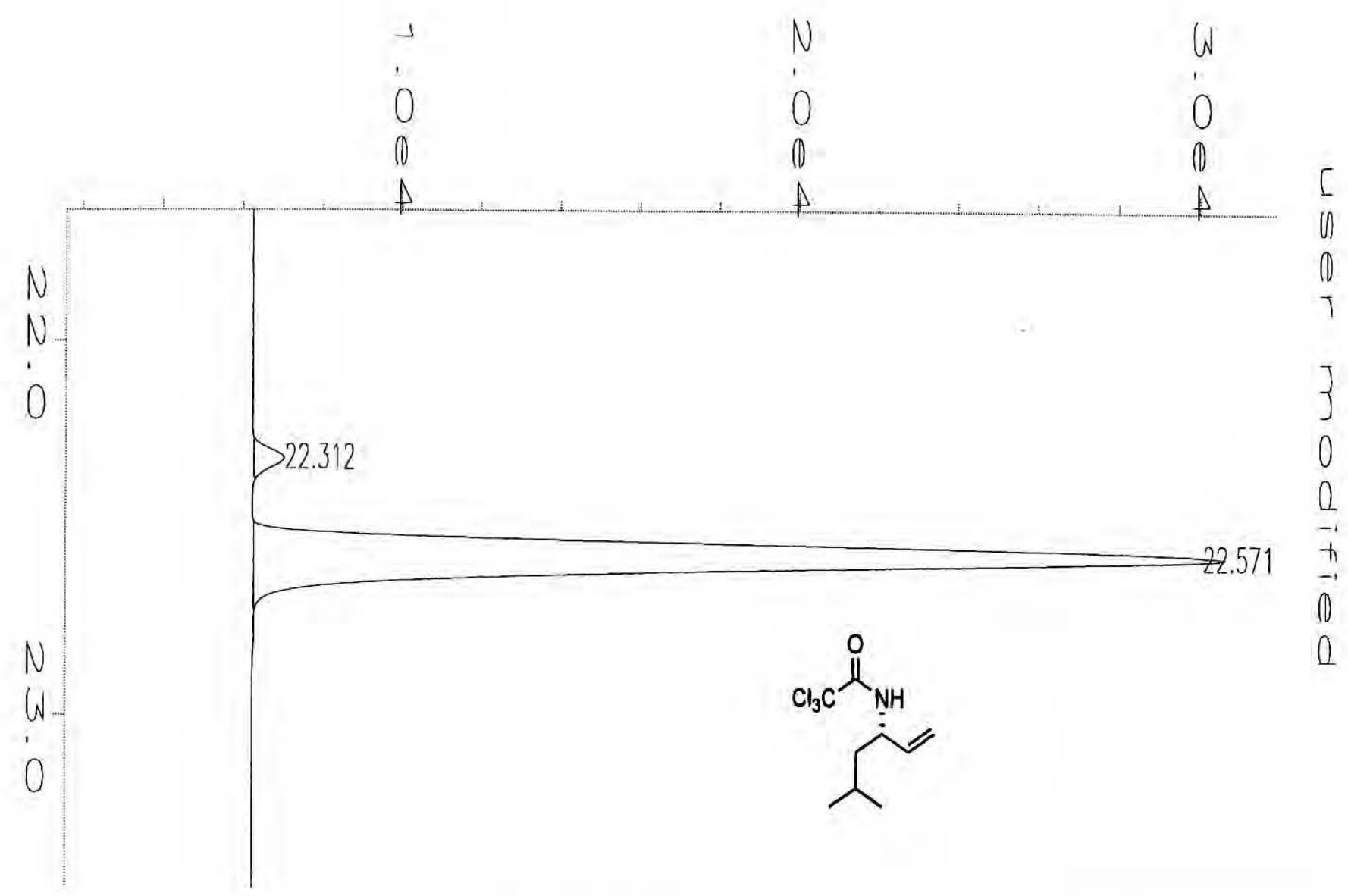

Area Percent Report

\begin{tabular}{|c|c|c|}
\hline erator & STEFAN & Page Number \\
\hline Instrument & INSTRUMEN & Vial Number \\
\hline $\begin{array}{l}\text { Sample Name } \\
\text { Run Time Bar Code }\end{array}$ & SK247//A & $\begin{array}{l}\text { Injection Number } \\
\text { Sequence Line }\end{array}$ \\
\hline
\end{tabular}

Report Created on: 26 Jun 04 12:57 PM

Instrument Method: LONGRUN.MTH Analysis Method : LONGRUN.MTH

Sig. 2 in $C: \backslash$ HPCHEM $\backslash 2 \backslash$ DATA $\backslash S T E F A N \backslash S K 247 B-Y . D$

\begin{tabular}{|ccccccc} 
Pk\# & Ret Time & Area & Height & Type & Width & Area $\%$ \\
\hline 1 & 22.312 & 2559 & 760 & MM & 0.056 & 2.2681 \\
2 & 22.571 & 110282 & 24363 & MM & 0.075 & 97.7319
\end{tabular}

Total area $=112841$

User Modified

(Chiraldex Gamma Cyclodextrin Trifluoroacetyl, $20 \mathrm{~m} \times 0.25 \mathrm{~mm}$; initial temperature: $50^{\circ} \mathrm{C}$, final temperature: $\left.150^{\circ} \mathrm{C}, 5^{\circ} \mathrm{C} / \mathrm{min}\right)$ 


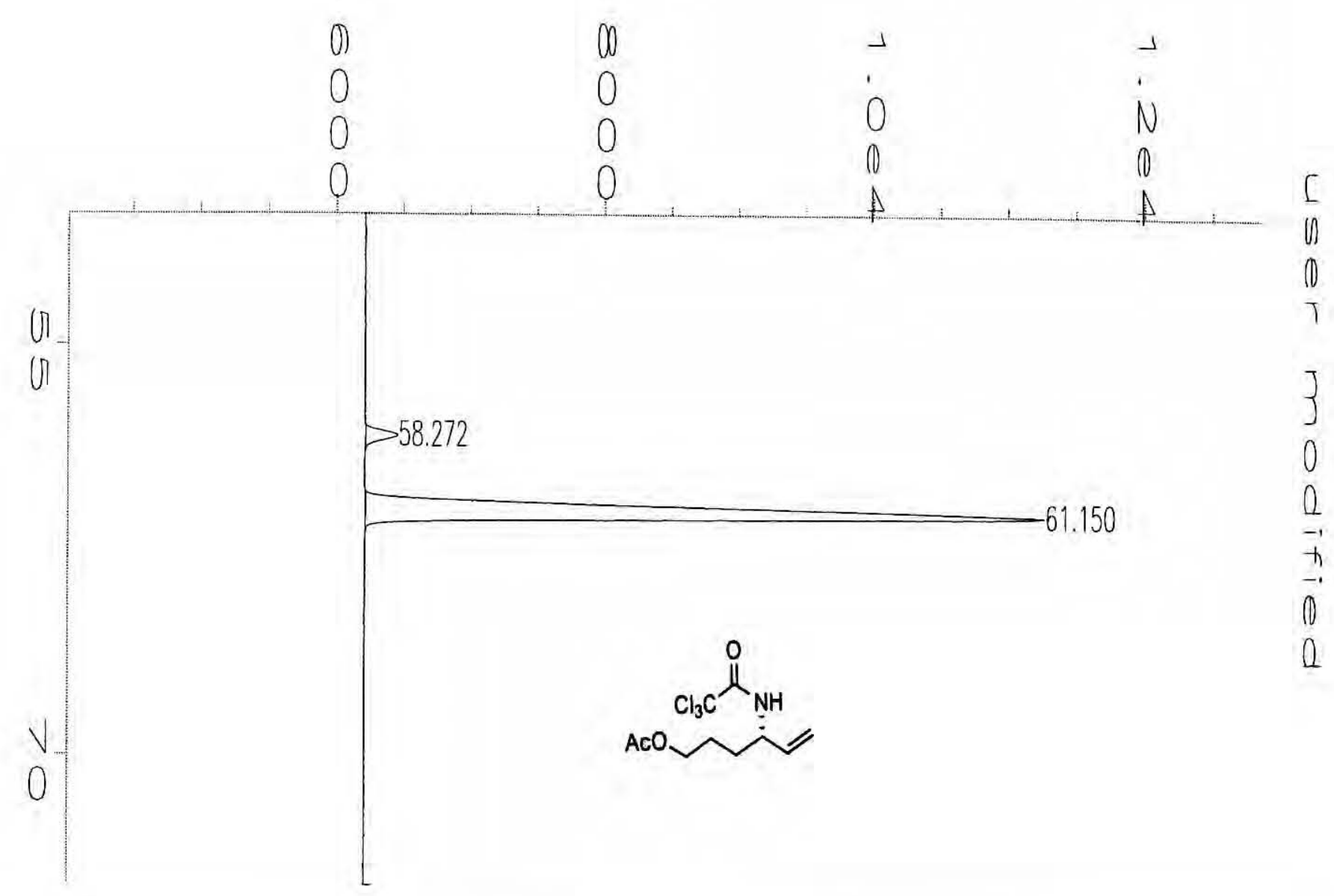

Area Percent Report

Data File Name : C: \HPCHEM \2\DATA \STEFAN \SK164A.D

Operator

Instrument

Sample Name

Run Time Bar Code:

Acquired on : 25 Mar 04 11:46 AM

Report Created on: 25 Mar 04 02:47 PM
Page Number : 1

Vial Number :

Injection Number :

Sequence Line :

Instrument Method: LONGRUN.MTH Analysis Method : LONGRUN.MTH

Sig. 2 in C: $\backslash$ HPCHEM $\backslash 2 \backslash$ DATA \STEFAN \SK164A.D

\begin{tabular}{|ccccccc} 
Pk\# & Ret Time & Area & Height & Type & width & Area $\%$ \\
\hline 1 & 58.272 & 5480 & 239 & BB & 0.290 & 3.6938 \\
2 & 61.150 & 142865 & 5052 & BB & 0.340 & 96.3062
\end{tabular}

Total area $=148345$

User Modified

(Chiraldex Gamma Cyclodextrin Trifluoroacetyl, $20 \mathrm{~m} \times 0.25 \mathrm{~mm}$; initial temperature: $50^{\circ} \mathrm{C}$, final temperature: $150^{\circ} \mathrm{C}, 5^{\circ} \mathrm{C} / \mathrm{min}$ ) 

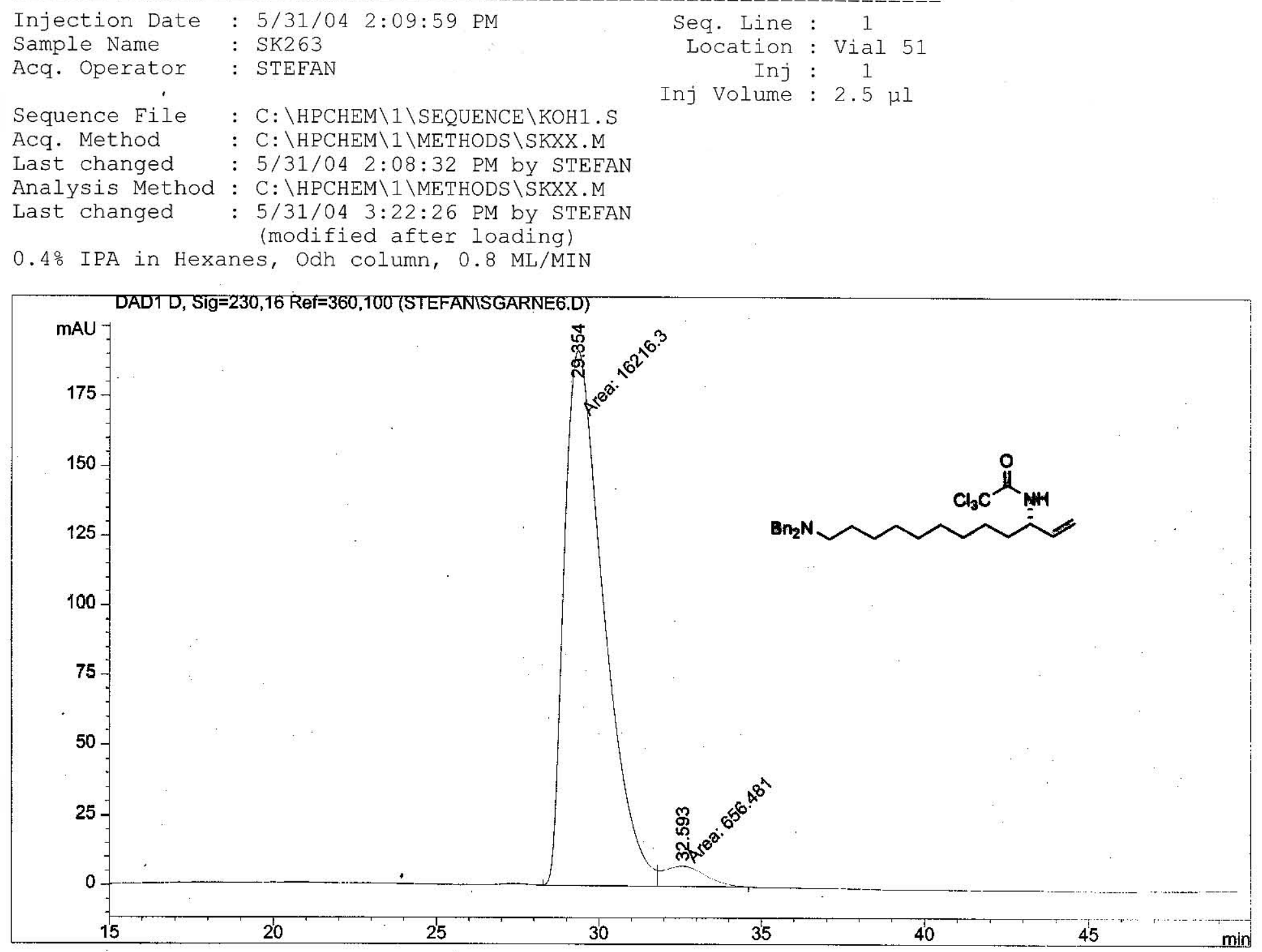

Area Percent Report

$\begin{array}{lll}\text { Sorted By } & : & \text { Signal } \\ \text { Multiplier } & : & 1.0000 \\ \text { Dilution } & : & 1.0000\end{array}$

Use Multiplier \& Dilution Factor with ISTDs

Signal 1: DAD1 D, Sig=230,16 Ref=360,100

\begin{tabular}{|c|c|c|c|c|c|c|}
\hline $\begin{array}{c}\text { Peak } \\
\#\end{array}$ & $\begin{array}{c}\text { RetTime } \\
\text { [min]. }\end{array}$ & Type & $\begin{array}{l}\text { Width } \\
\text { [min] }\end{array}$ & $\begin{array}{c}\text { Area } \\
{\left[\mathrm{mAU}{ }^{*} \mathrm{~s}\right]}\end{array}$ & $\begin{array}{l}\text { Height } \\
{[\mathrm{mAU}]}\end{array}$ & $\begin{array}{c}\text { Area } \\
\frac{\circ}{\delta}\end{array}$ \\
\hline-- & & & & ------- & 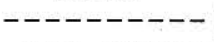 & \\
\hline 1 & 29.354 & MF & 1.4105 & $1.62163 \mathrm{e} 4$ & 191.62035 & 96.1092 \\
\hline 2 & 32. & EM & 1.4944 & 656.48114 & 7.32178 & 8908 \\
\hline
\end{tabular}

Totals :

$$
1.68728 \mathrm{e} 4 \quad 198.94212
$$

Results obtained with enhanced integrator!

\section{Summed Peaks Report}

Signal 1: DAD1 D, Sig=230,16 Ref=360,100 
4\% IPA IN HEXANES, $0.8 \mathrm{MI} / \mathrm{MIN}, \mathrm{ODH}$, no guard

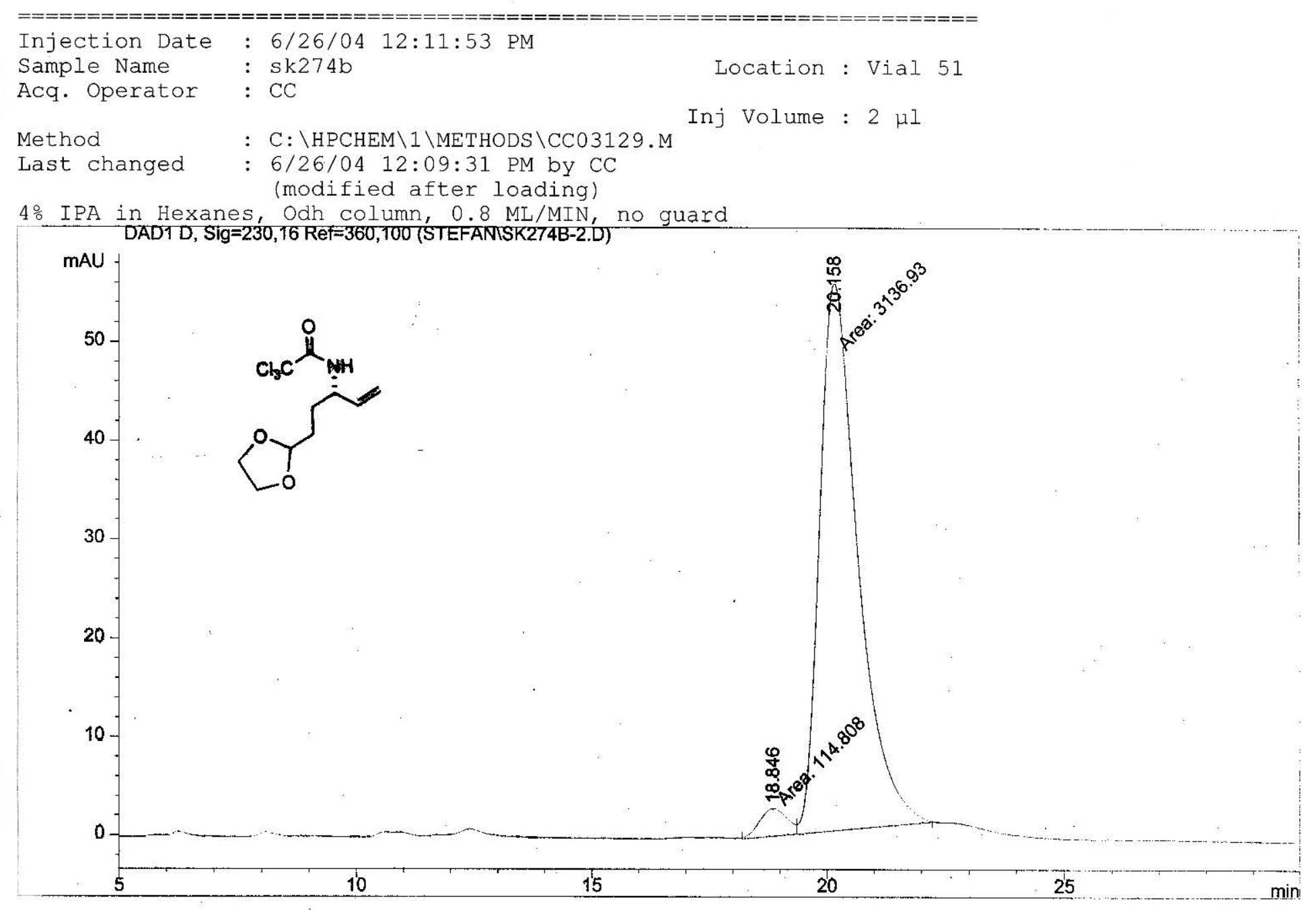

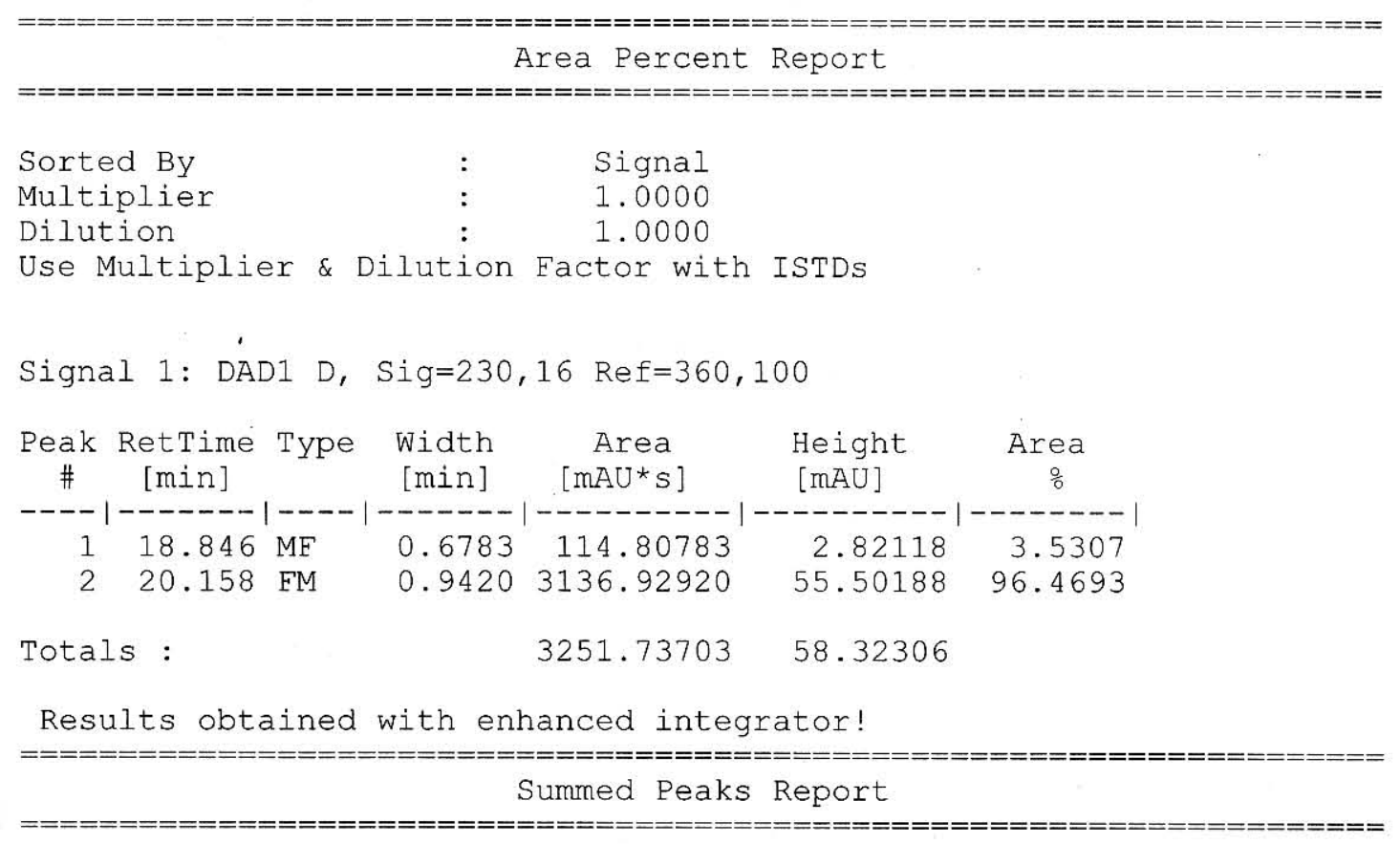

Signal 1: DAD1 D, Sig=230,16 Ref=360,100 


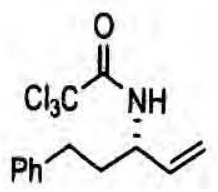

\section{sk 125}

$1 \mathrm{H}$ spectrum
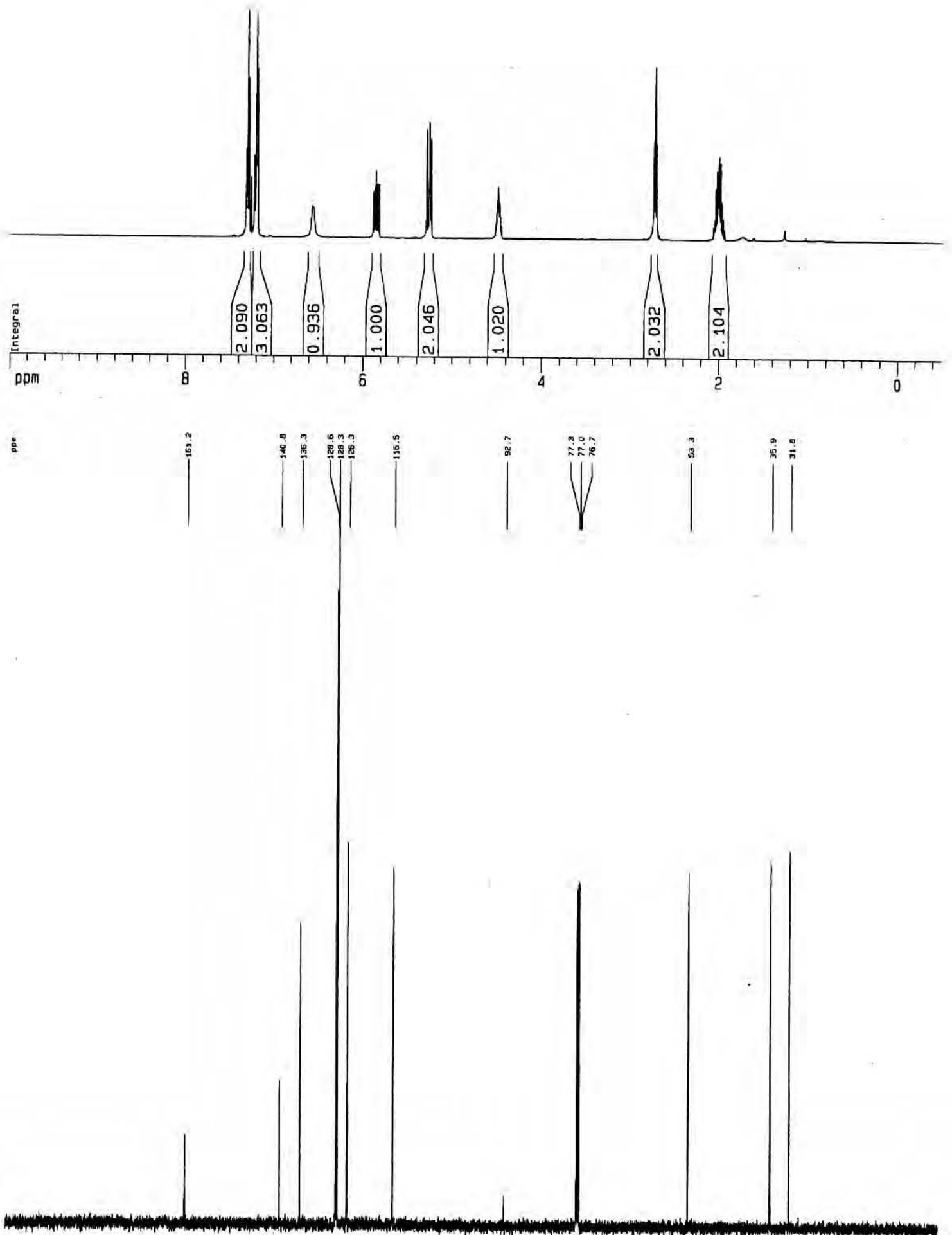

ppm

150 
<smiles>C=C[C@H](C)NC(=O)C(Cl)(Cl)Cl</smiles>

sk 157

$1 H$ spectrum

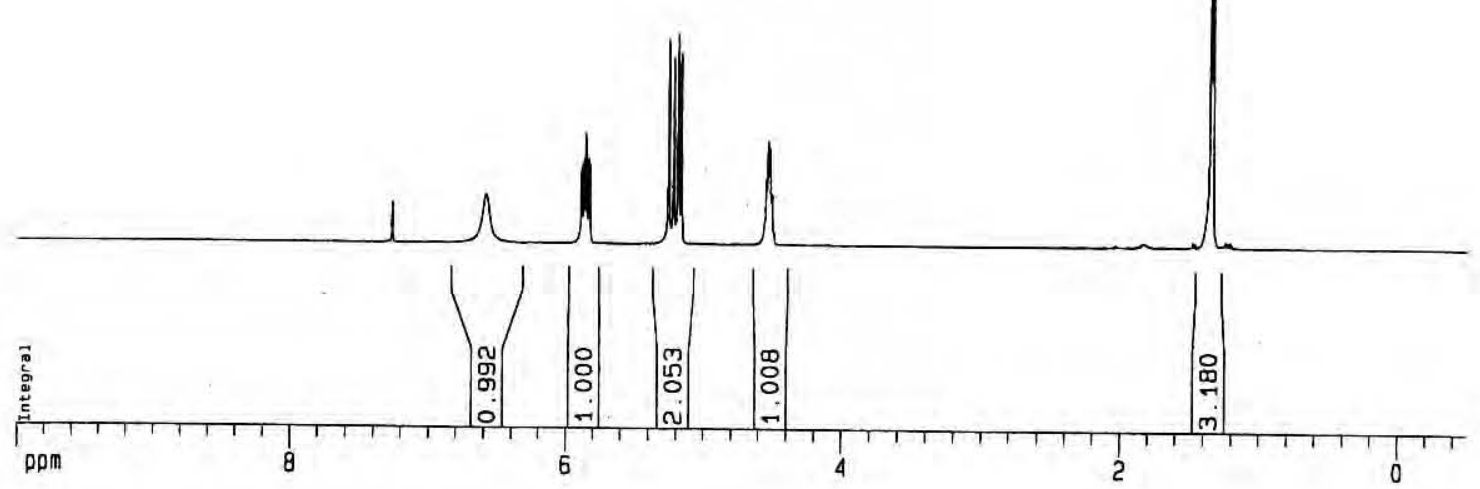

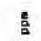
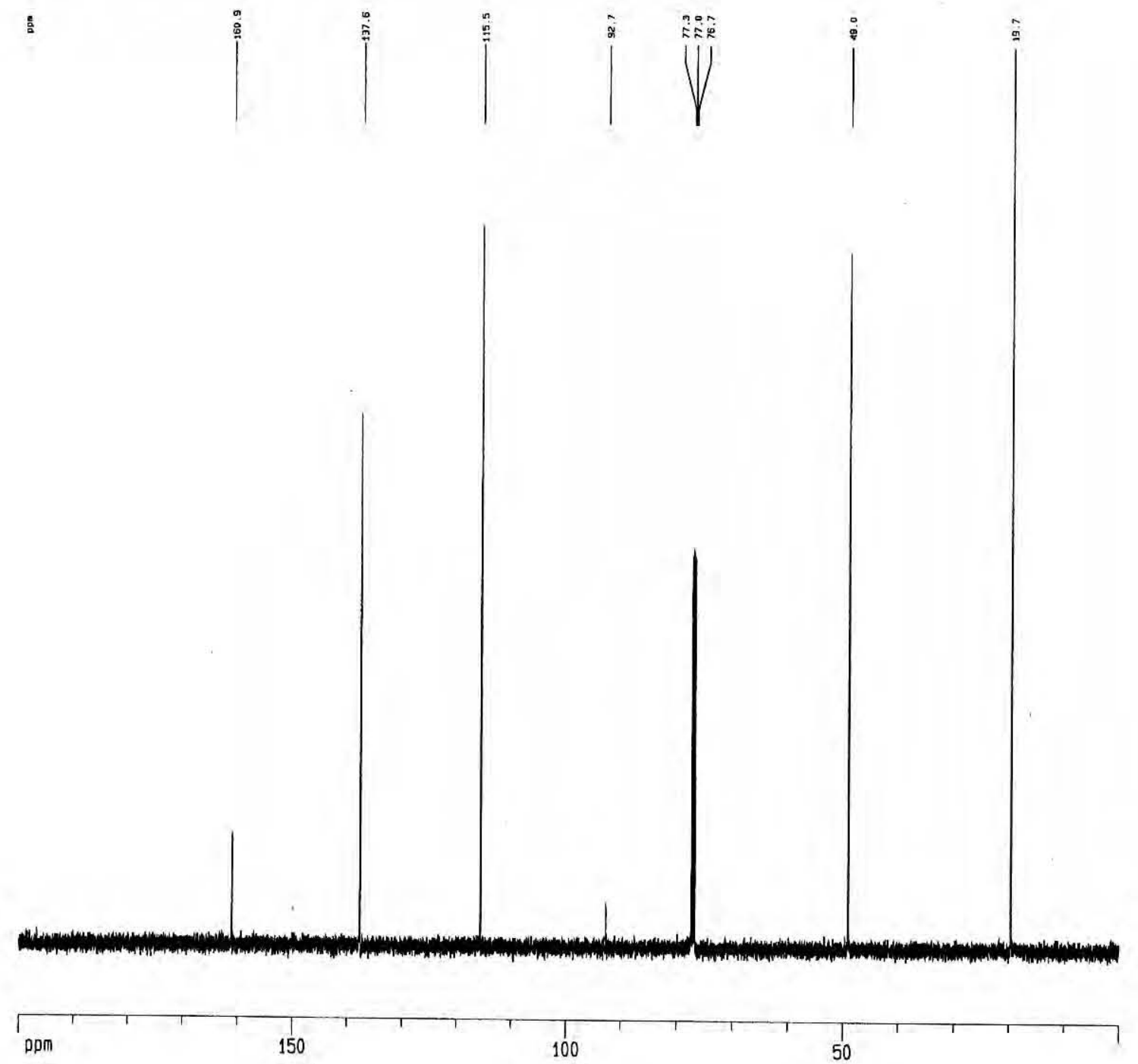
<smiles>C=CC(CCC)NC(=O)Cl</smiles>

5k162

$1 \mathrm{H}$ spectrum
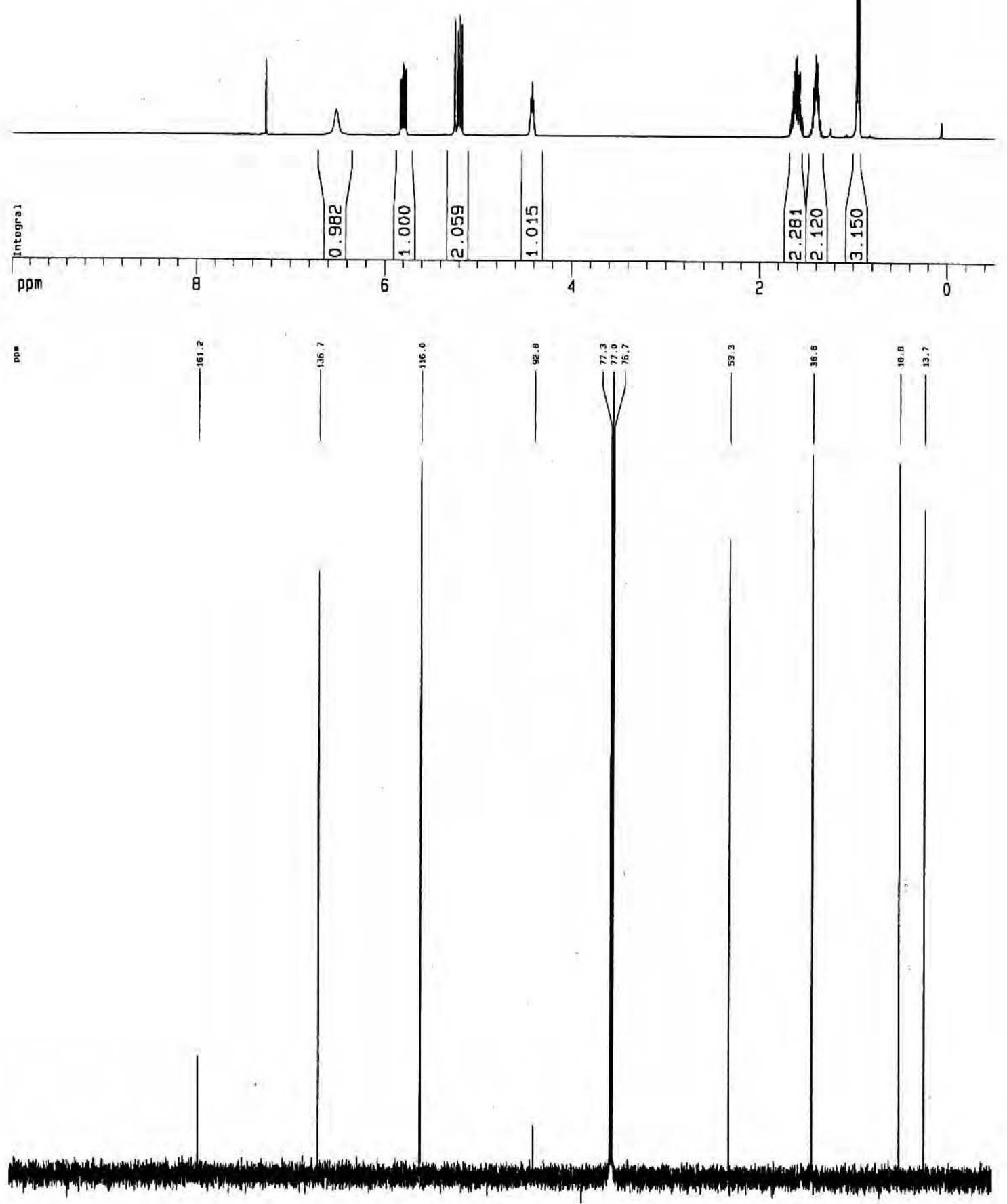

ppm 150 100 


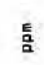
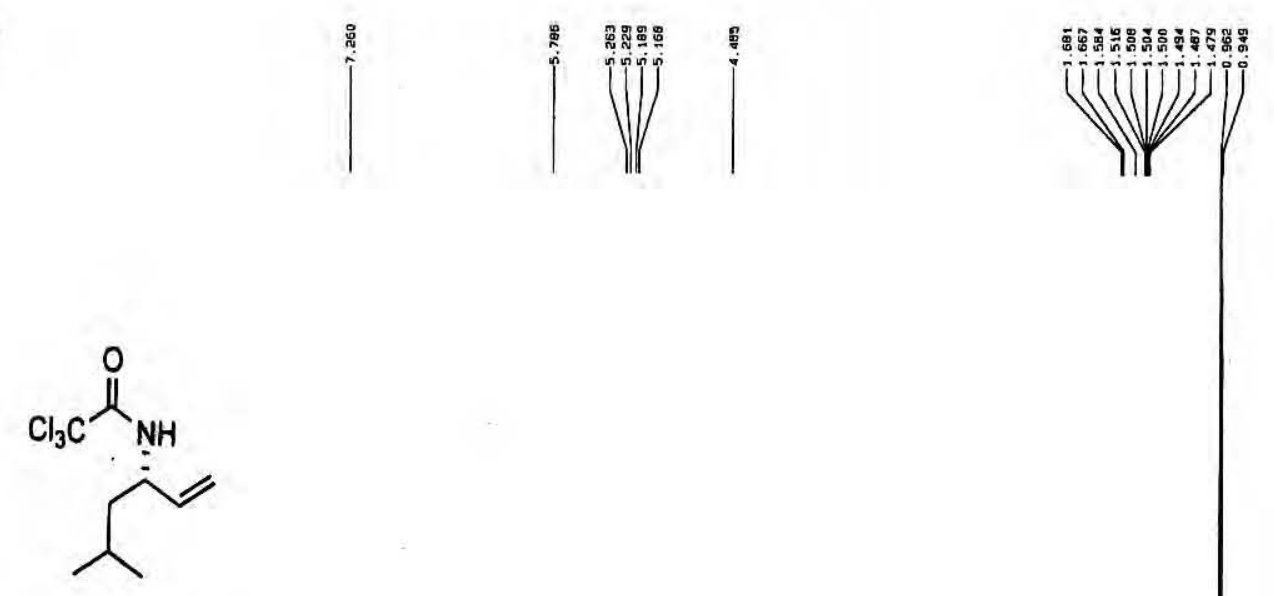

sk274A
$1 H$ spectrum
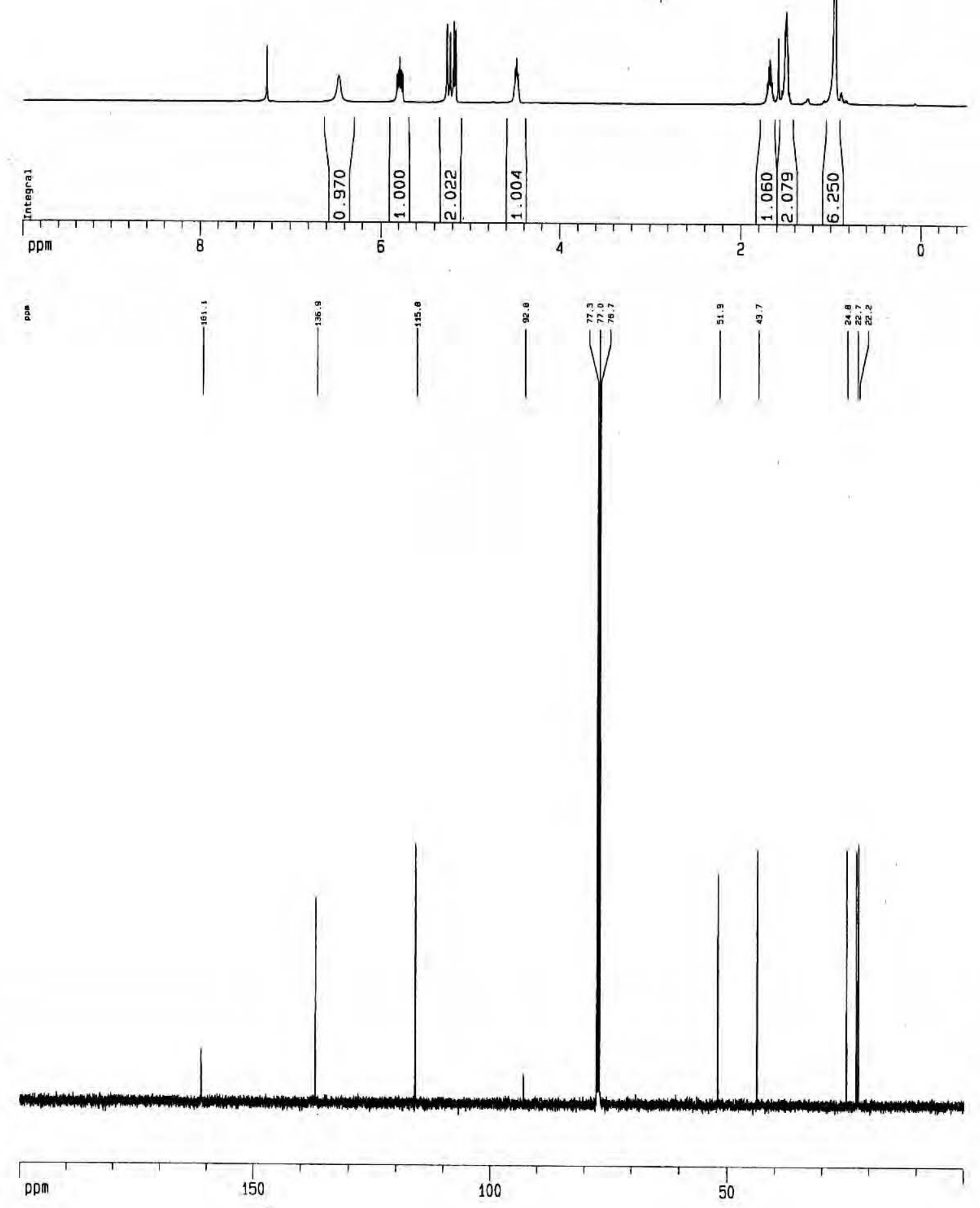

S12 
<smiles>CCC(CCCOC(C)(Cl)Cl)NC(=O)C(Cl)(Cl)Cl</smiles>

sk 164

$1 H$ spectrum

s
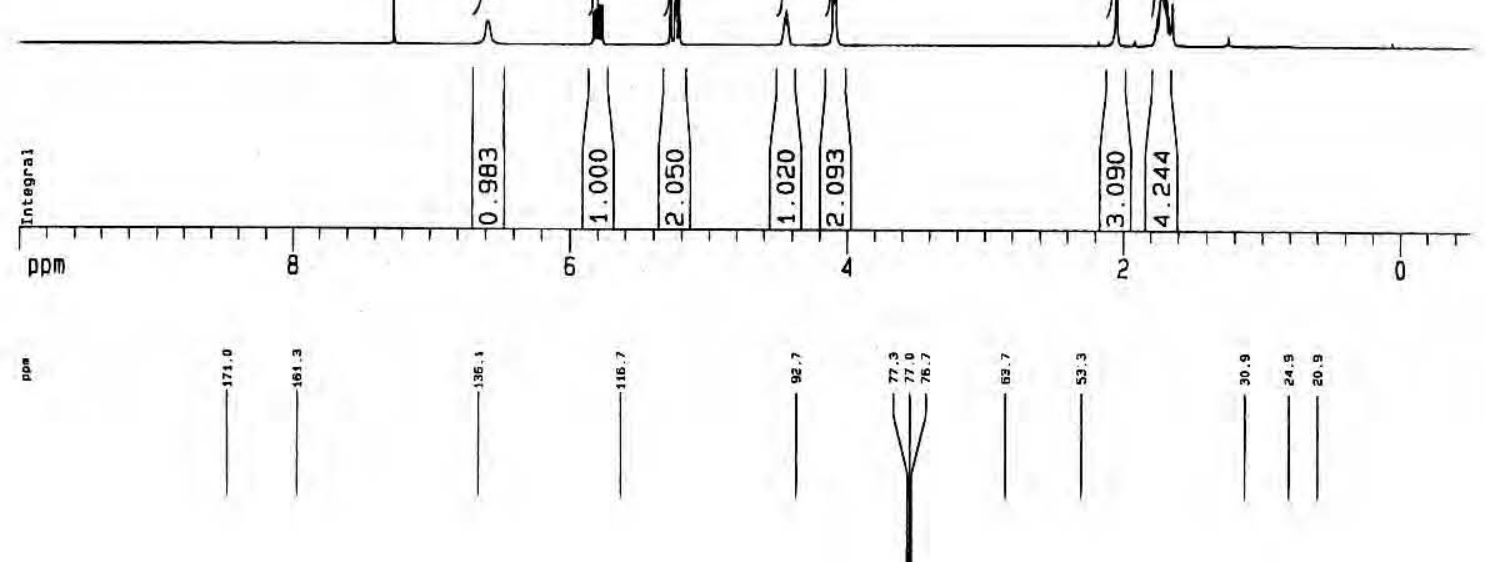

ppm

150

100

50 


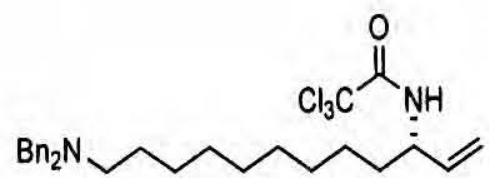

5k274 C

$1 \mathrm{H}$ spectrum
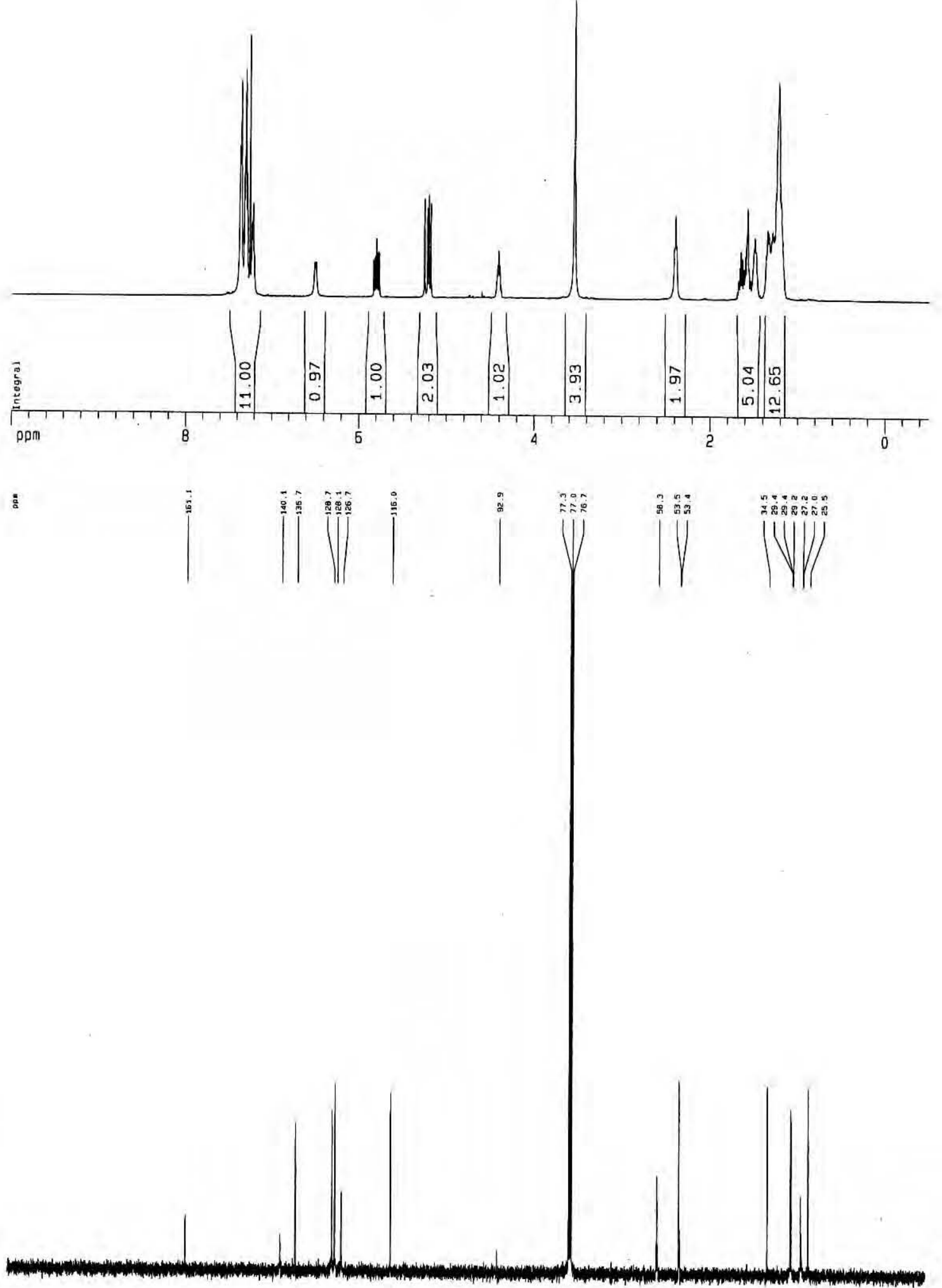

pp

150

50 

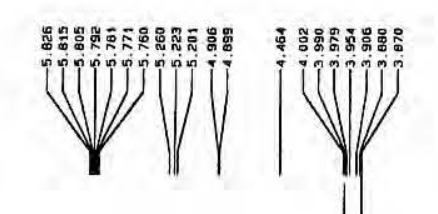

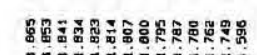

sk274b

$1 \mathrm{H}$ spectrum<smiles>C=CCC(CCC1OCCO1)NC(=O)C(Cl)(Cl)Cl</smiles>

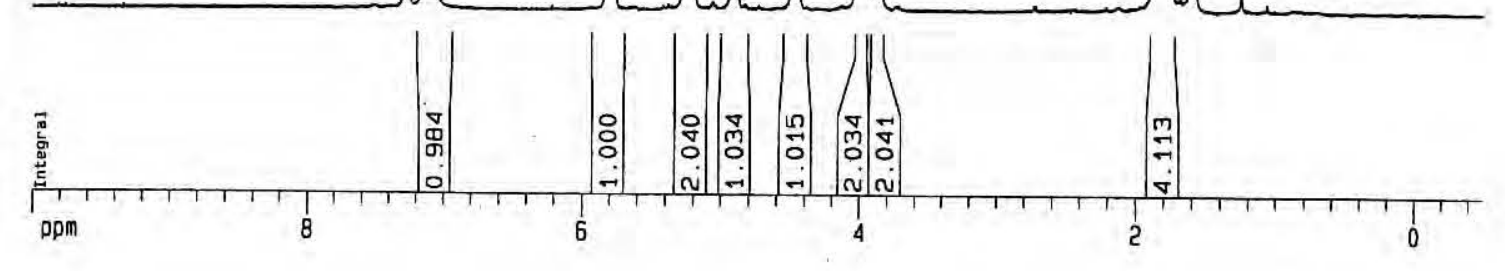

$₫$

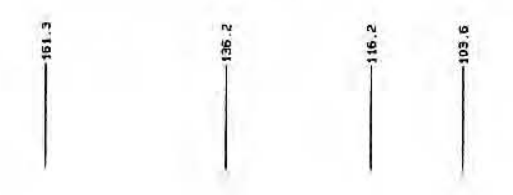

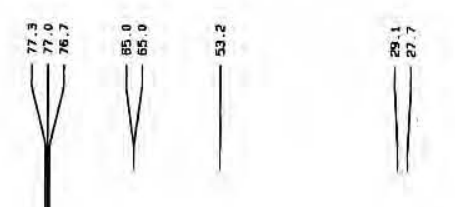

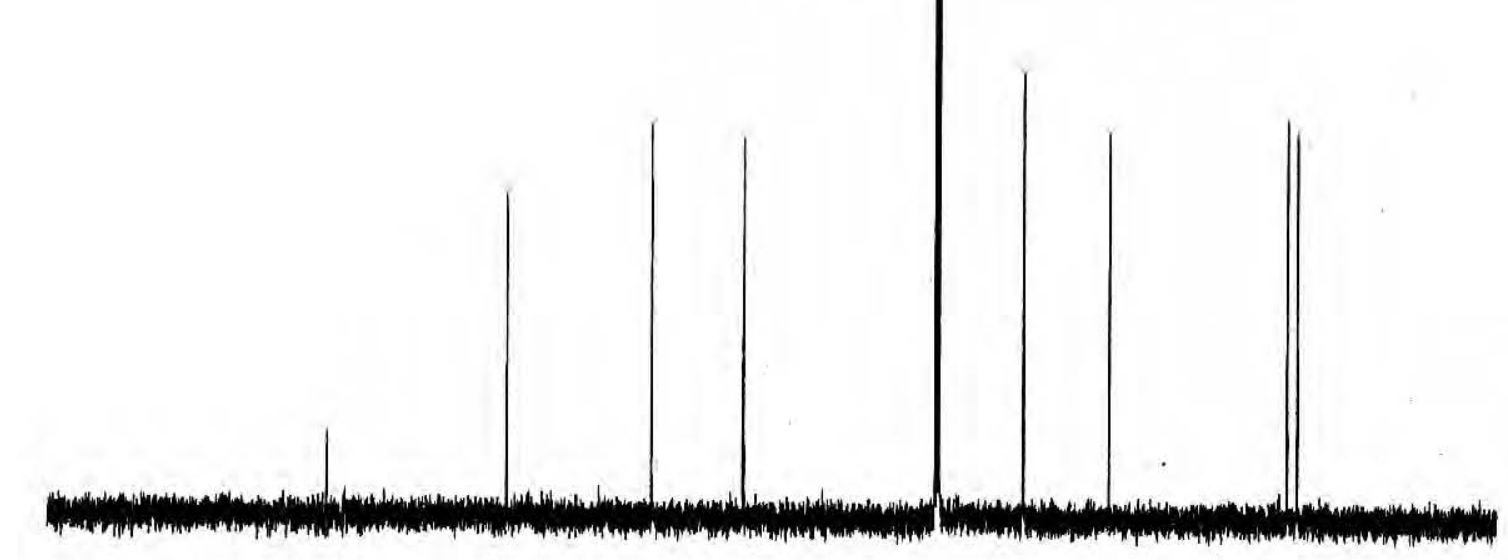

ppm

150

100

50 


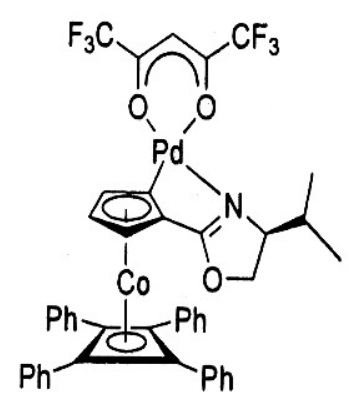

sk131

$1 \mathrm{H}$ spectrum
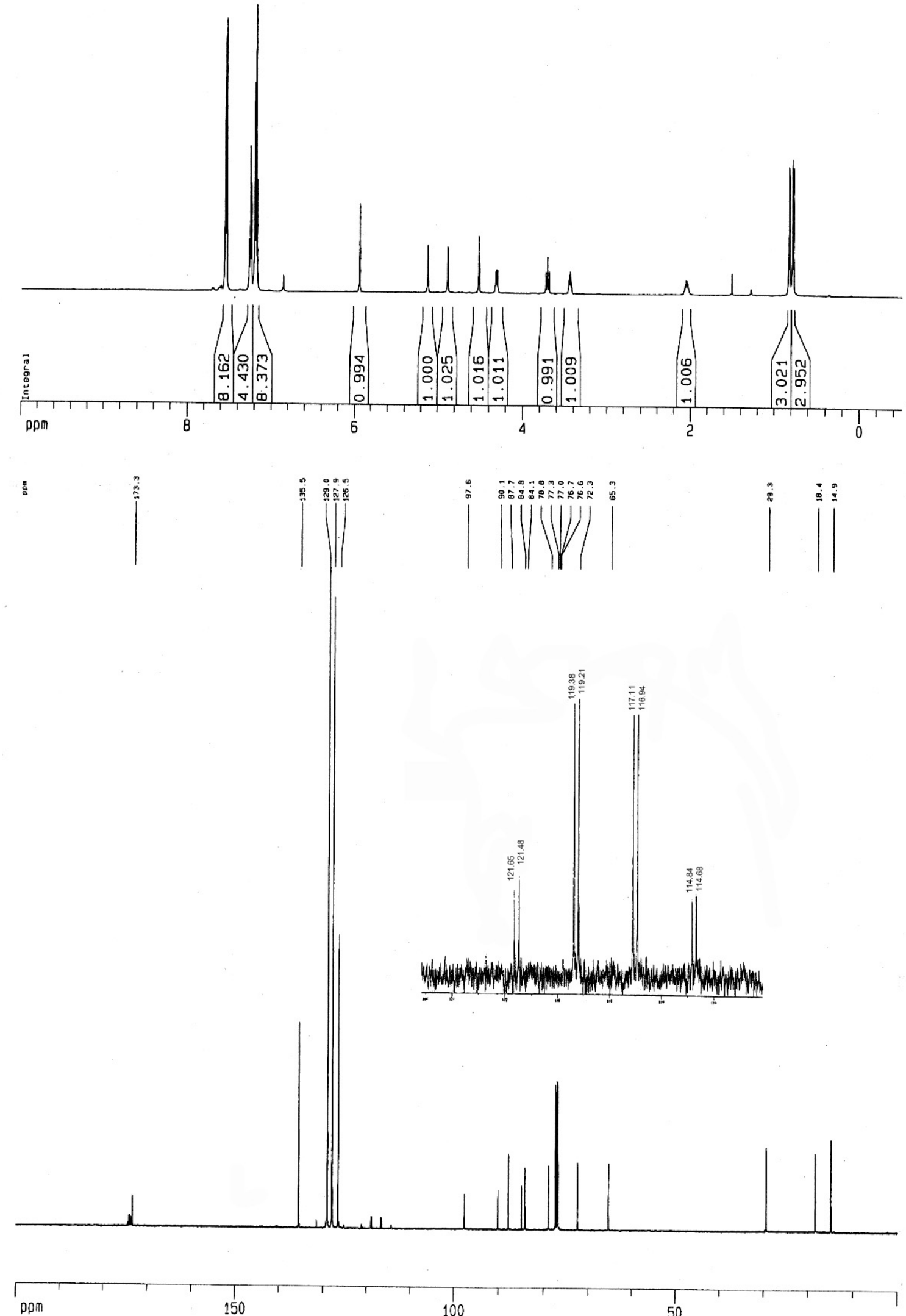

150

100

50 


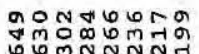

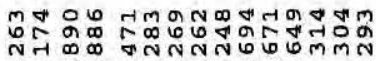

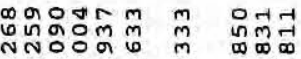

oranara

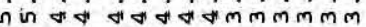

लंखितन ने 000

(V)

11

VIII
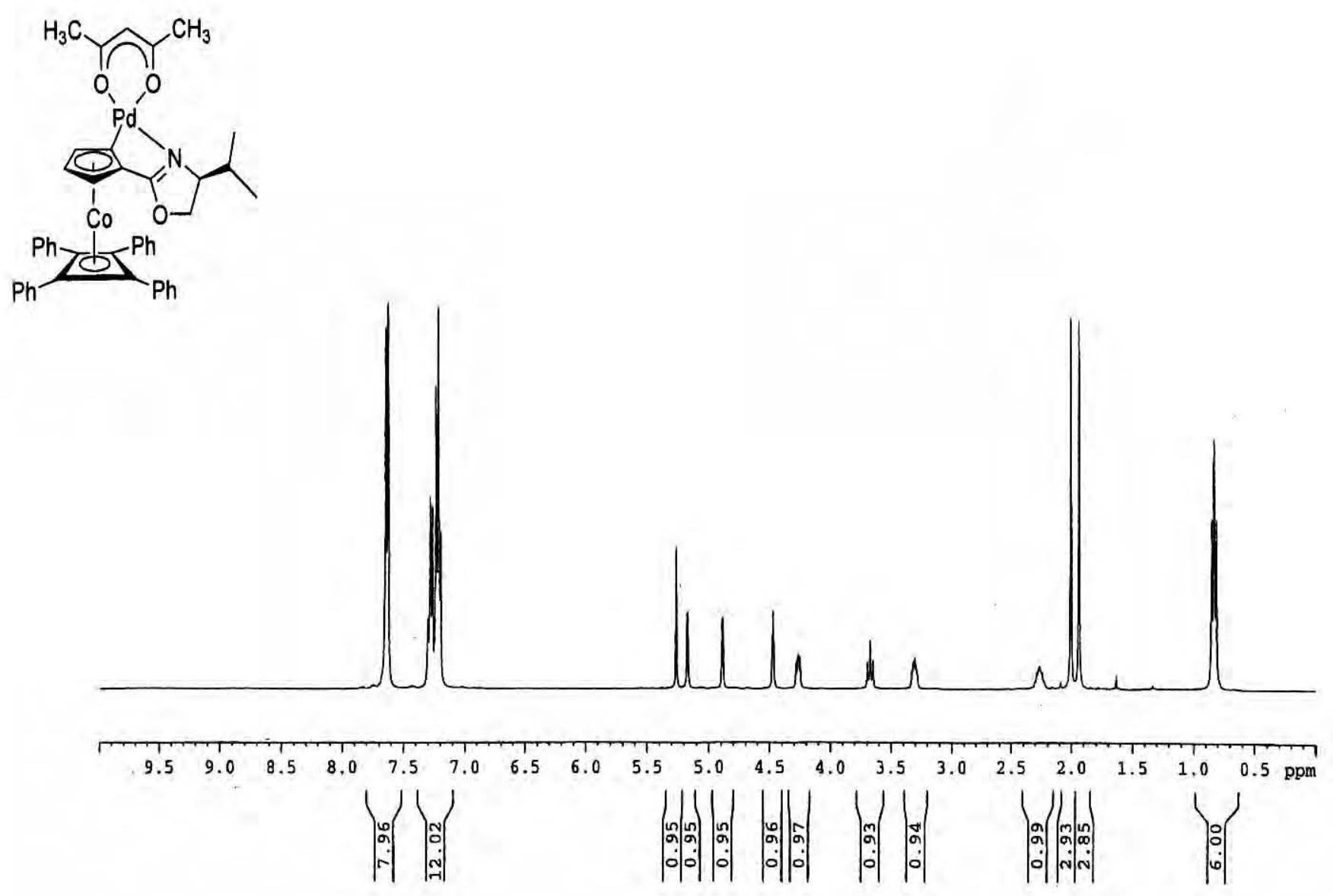

$\left.\right|^{\substack{n+\infty \\ 0}}$

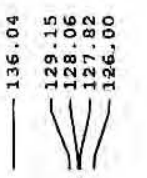

in

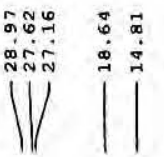

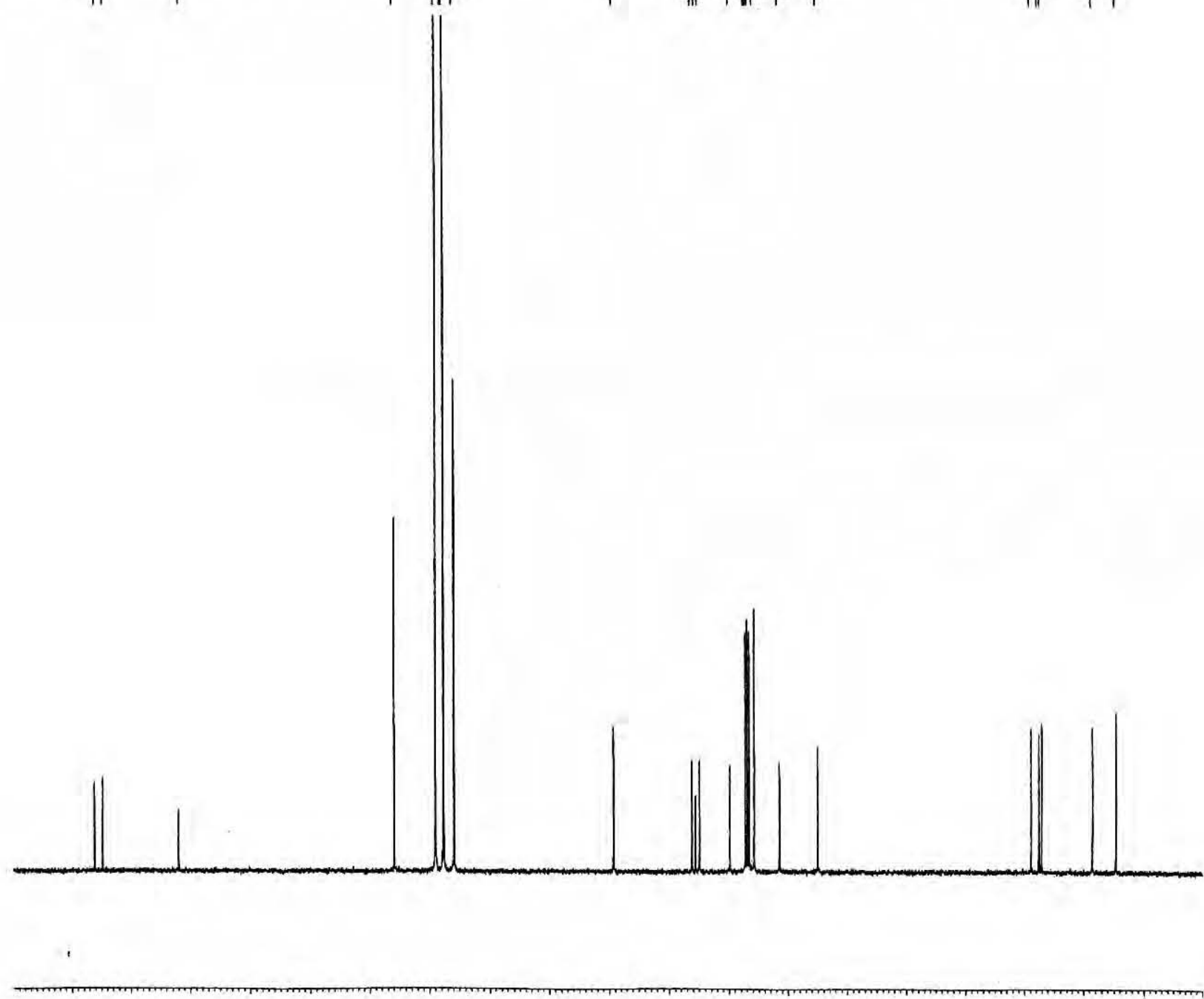

